\author{
Military Technical College \\ Kobry Elkobbah, \\ Cairo, Egypt
}

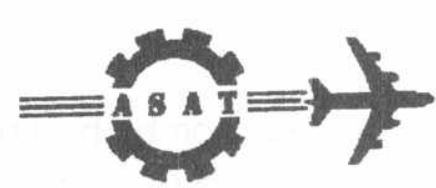

$8^{\text {th }}$ International Conference on Aerospace Sciences \&

Aviation Technology

\title{
SMART BOMB'S GUIDANCE LOOP DESIGN
}

\author{
Ahmed S. Gad Mohsen S. Aly ${ }^{*}$
}

\begin{abstract}
By progressing of time, the effectiveness and importance of smart weapons become more obvious. Among the wide varieties of known smart weapons, guided bombs are of special interest since they enable precise destruction of enemy targets in quite safe manner. The smart bombs are formed by attaching seeker and control units to the conventional unguided bombs. The seeker type determines the type of the bomb. Television (TV), millimeter waves, laser, or infrared seekers may be employed. The seeker involves the target acquisition and tracking systems. It provides the bomb-target line-of-sight signal to the guidance section which in turn produce the guidance commands to the control unit. The control unit contains the control fins and the necessary subsystems for the instantaneous execution of the guidance commands.
\end{abstract}

In this paper, attention will be paid to the design of the bomb guidance loop. The design process passes through two steps. In the first step, an identification to the bomb airframe is carried out. The nonlinear flight dynamical behavior of the airframe is linearized around a chosen trim operating point. The identification is made via a six-degrees-of-freedom (6-DOF) flight model of the bomb, wherefrom the transient response of the airframe is computed. Out of this step, the transfer function of the bomb airframe (that relates the airframe maneuver to the control fins deflection) is obtained. In the second step, the guidance loop is analyzed. First, Ideal (inertialess) and real (with finite inertia) seekers are considered. In view of the problem kinematics, the guidance loop stability can not be maintained throughout the flight. The design criterion is that the stability of the guidance loop is maintained over most the entire bomb flight trajectory. It is shown that this can be achieved via a proportional-plus-differential (PD) controller that is placed in the bomb board.

In this paper, noise free system is considered as a primary step in the design. However, the effect of inherent noises and external disturbances can be studied by inserting them as additional inputs to the seeker, and this is beyond the scope of the present paper.

* Ass. Lecturer, Radar and Guidance Dpt. , Military Technical College, Cairo, Egypt.

*A Ass. Prof., Radar and Guidance Dpt., Military Technical College, Cairo, Egypt. 


\section{INTRODUCTION:}

Although many types of guided bombs had been developed, only two types are proven to be effective in the operational use, [1]. These are the laser guided and electro-optical or TV guided "glide" bombs. They incorporate either a TV vidicon or a laser receiver and guidance and flight control system to accurately move the bomb control fins. TV guided bomb is equipped with a small vidicon which allows the pilot or co-pilot to monitor the target on a master TV screen in the cockpit. Once he has located a target in a standard visual manner, he shifts his view to the TV screen and using a hand control unit, centers the TV camera cross-hair's sights on the target. The pilot then activates a lock-on and releases the bomb which operates on its own and is guided automatically to the point on which its TV seeker has been locked. TV guided weapons are limited mostly to daylight hours, and good weather. Targets must have a high optical contrast for the TV camera to home-in. They can also be equipped with a TV data link, that displays in the cockpit exactly what the camera in the bomb sees. This allows the pilot to remotely guide the bomb to the target if the need arises, [2,3].

Laser guided bombs are more frequently used against targets of opportunity, such as tanks, or other moving targets. Laser guided bombs are also used successfully against oil fields and power plants. TV guided bombs, on the other hand, due to their glide capability, are more suitable against targets presented in the vertical plane, such as railroad tunnels and caves. They are used where designation is not possible and targets have sufficient contrast. Since a bridge and river background present good contrast, TV guided bombs as well as laser guided bombs have been used against bridges.

The TV guided bomb has an edge in this respect because the laser bomb must be delivered very accurately within certain "basket" limitations, depending on the reflected laser beam. It is quite possible that a pilot could overshoot the "basket", if he goes too fast or approaches at an improper angle. Also, since laser guided bombs sometimes require a separate "spotter" plane, the chances of loss are increased. The size of the reflected laser beam or "basket" largely depends on the target itself and its optical surface qualities. If the target surface is diffusive, it will more widely scatter the laser beam and provide a bigger "basket". On the other hand, an optically smooth target would more closely reflect the incident laser beam and the "basket" would be considerably smaller. Of course, the pilot never sees the actual reflected laser energy. A laser seeker and display indicates to him when he is in the "basket area" and when to release the laser bomb.

There are two main advantages to the use of guided bombs. One is the lower overall cost, since fewer bombs are needed to hit the target. The second is that delivery aircraft is not locked into any prescribed flight path and can take evasive action at any time during the delivery run without affecting weapon accuracy. Two basic modes of delivery are used with TV guided bombs, direct and indirect. For direct attack, the bomb is locked onto the target before launch and flies autoriomously to impact. For indirect attack, the bomb flies a programmed midcourse climb-plus-glide phase that extends stand-off capability to enhance aircraft 
survivability. Midcourse guidance updates are provided by means of the data link. When the target becomes into view after launch, the bomb system operator may elect to lock the electronic tracker on the target for automatic terminal homing, manually update the automatic track aim-point, or manually guide the bomb to target impact via the data link. The primary mode of operation is indirect attack.

In this paper, the TV guided bomb launched in the indirect attack mode is considered. The objective is to investigate the homing guidance system performance of the bomb. Thus, the nonlinear aerodynamic coefficients of the bomb are calculated, the 6-DOF simulation program is developed, and the linearized aerodynamic transfer functions of the bomb is obtained. The guidance loop of the bomb is designed and the stability of the loop is tested. The system is found conditionally stable when using ideal homing head and has acceptable missdistance and minimum stable LOS range. Using homing head with finite lags, makes the designed loop unstable and gives large missdistance. Adding PD controller in the forward path of the guidance loop, adds a zero to the left hand side of the imaginary axis in the root locus plot and pulls the root loci to the left. This improves the system stability and fulfills the desired design goals. This improvement is evaluated throughout the root locus plot, the system response, the bomb trajectory, and the missdistance, [4].

\section{CALCULATION OF THE LINEARIZED AERODYNAMIC TRANSFER FUNCTIONS}

The aerodynamic shape, axes, forces, moments, velocities, and angles are shown in fig. 1 and fig. 2, [5].

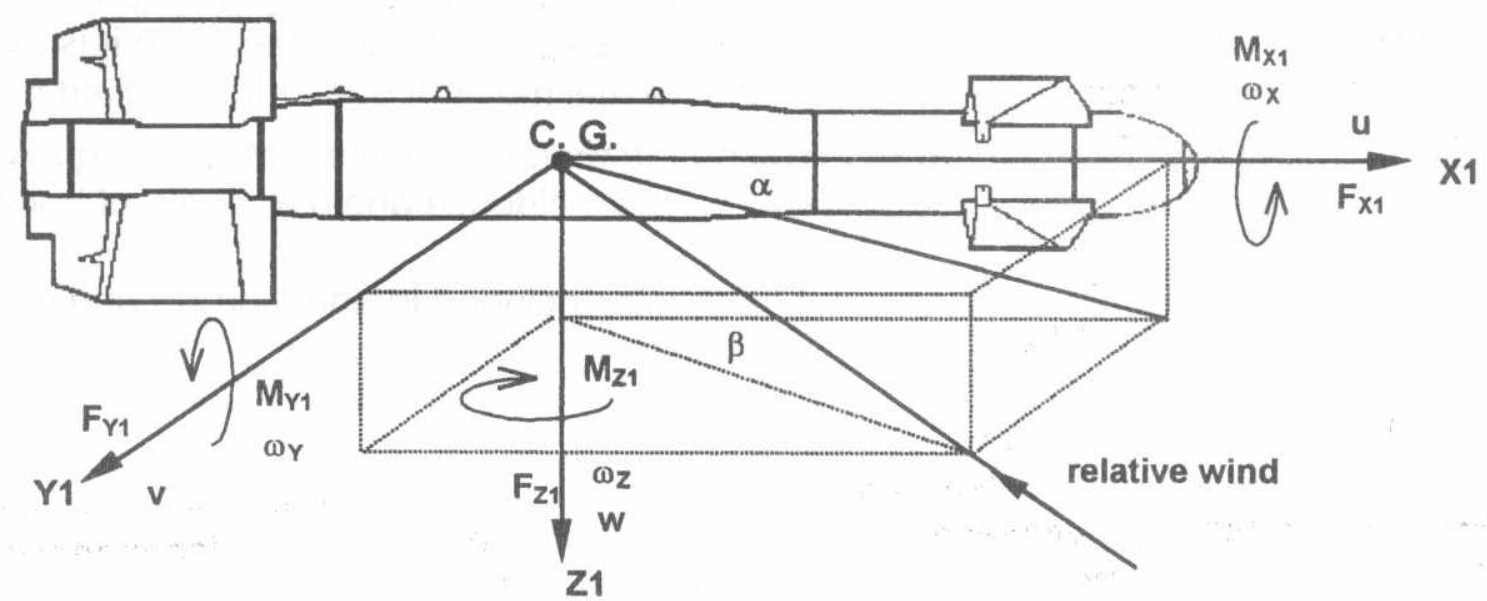

Fig. 1 Aerodynamic shape, axes, forces, moments, velocities, and angles 


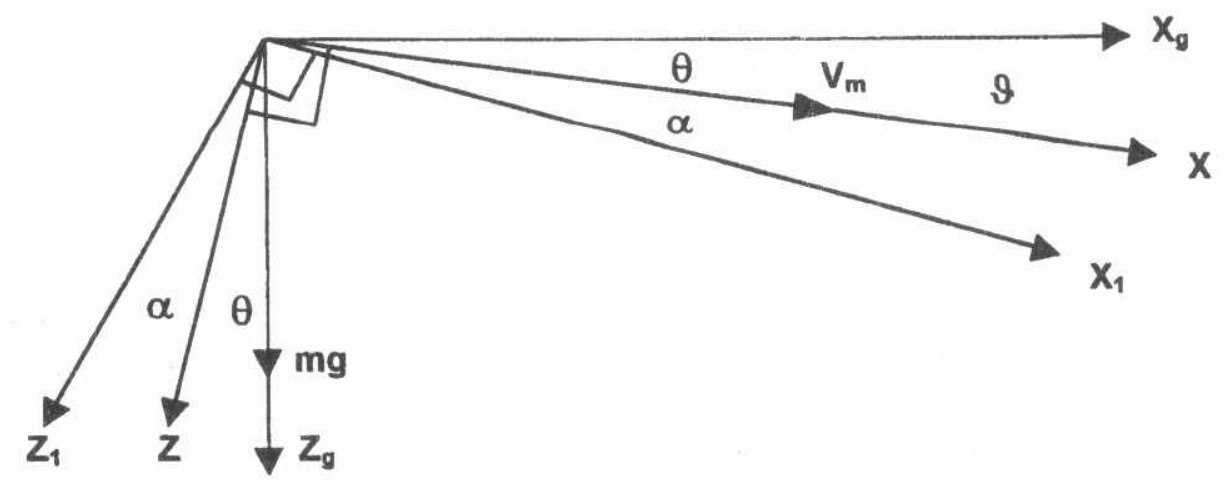

Fig. 2 The kinematic relation between $\alpha, \vartheta$, and $\theta$ in vertical plane

The system of equations describing the vertical plane motion can be written according to the following equations, $[5,6]$ :

$$
\begin{aligned}
& \sum \text { Forces }=\frac{d}{d t}\left(m \vec{V}_{m}\right), \\
& m V_{m} \dot{\theta}+m g \cos \theta=F_{z 1}(\alpha)+F_{z 1}(\delta), \\
& \sum \text { Moments }=\frac{d}{d t}(I \vec{\omega}), \\
& \dot{\omega}_{y} I_{y y}=M_{y 1}(\alpha)+M_{y 1}(\delta)+M_{y 1}\left(\omega_{y}\right),
\end{aligned}
$$

and $\alpha=\vartheta-\theta$.

Where $F_{Z_{1}}(\alpha)$ is the lift force due to incidence $\alpha, F_{z_{1}}(\delta)$ is the lift force due to control fin deflection $\delta, I_{y y}$ is the moment of inertia of bomb around $y$-axis, $\dot{\omega}_{y}$ is the angular acceleration of bomb around $y$-axis, $M_{y 1}(\alpha)$ is the moment around $y$-axis due to incidence, $M_{y 1}(\delta)$ is the moment around $y$-axis due to control fin deflection $\delta, M_{y 1}\left(\omega_{y}\right)$ is the moment around $y$-axis due to angुular movement $\omega_{y}$.

Equation (2) describes the equilibrium of forces, along the (pitch) Z-axis. Equation (4) describes the equilibrium of moments around the $y$-axis, so it is also the rotation in the vertical plane. $\dot{\omega}_{y} I_{y y}$ is the inertial moment due to the applied moment $M_{y_{1}}(\delta)$, the stabilizing moment $M_{y_{1}}(\alpha)$, and the damping moment $M_{y_{1}}\left(\omega_{y}\right)$. Equation (5) describes the kinematic relation between the incidence angle $\alpha$, angle of bomb axis $\vartheta$, and angle of flight path $\theta$.

The aerodynamic for'ses and moments, included in equation (2) and equation (4) could be written as:

$$
\begin{aligned}
& F_{z_{1}}(\alpha)=C_{z}(\alpha) S q, \\
& F_{z 1}(\delta)=C_{z}(\delta) S q \\
& M_{y 1}(\alpha)=m_{y}(\alpha) S q L_{f}, \\
& M_{y 1}(\delta)=m_{y}(\delta) S q L_{f} \\
& M_{y 1}\left(\omega_{y}\right)=m_{y}\left(\omega_{y}\right) S q L_{1}{ }^{2} N_{m} .
\end{aligned}
$$

Where $C_{z}$ and $m_{y}$ are dimensionless coefficients of lifting forces and moments. These aerodynamic coefficients are fully expressing the nonlinear dependence of lifting forces and moments upon the incidence angle $\alpha$ and control fin deflection. Moreover, they are functions of flight velocity. 
Equations (2) to (10) are nonlinear due to the presence of the nonlinear sinusoidal functions and the nonlinear behavior of the aerodynamic coefficients. The first assumption is that the incidence angle $\alpha$ is so small such that:

$\sin \alpha=\alpha$ and $\cos \alpha=1$.

As well, the aerodynamic coefficients can be represented by their derivatives viz.

$$
\begin{aligned}
& C_{z}(\alpha)=C_{z}{ }^{\alpha} \alpha, \\
& m_{y}(\alpha)=m_{y}{ }^{\alpha} \alpha .
\end{aligned}
$$

Where $\mathrm{C}_{z}{ }^{\alpha}$ and $\mathrm{m}_{\mathrm{y}}{ }^{\alpha}$ are the slopes of the lift coefficient and moment coefficient curves, respectively.

The second assumption is that the control fin deflection and body turn rate are small such that:

$$
\begin{aligned}
& \mathrm{C}_{z}(\delta)=\mathrm{C}_{z}{ }^{\delta} \delta \\
& \mathrm{m}_{\mathrm{y}}(\delta)=\mathrm{m}_{\mathrm{y}}{ }^{\delta} \delta \\
& \text { and } \mathrm{m}_{\mathrm{y}}\left(\omega_{\mathrm{y}}\right)=\mathrm{m}_{\mathrm{y}}{ }^{\omega \mathrm{y}} \omega_{\mathrm{y}} .
\end{aligned}
$$

These assumptions are quite accurate during the steady operation of the guidance loop. However, their validity during the transient periods will be checked as shown in the next section. The third assumption is omission of the gravity component $(m g \cos \theta)$. Numerical computations show that the value of this term is slowly varying during flight and thus can be considered as a constant bias usually called gravity bias. This bias will be considered after the design of the guidance loop as a constant fin deflection $d_{g}$ that compensate the gravity effect.

$$
\begin{aligned}
& \mathrm{mV}_{\mathrm{m}} \dot{\theta}=\mathrm{C}_{z}^{\alpha} \alpha \mathrm{Sq}+\mathrm{C}_{\mathrm{z}}^{\delta} \delta S q . \\
& \dot{\omega}_{\mathrm{y}} \mathrm{I}_{\mathrm{y} y}=\mathrm{m}_{\mathrm{y}}^{\delta} \delta S q \mathrm{~L}_{\mathrm{f}}+\mathrm{m}_{\mathrm{y}}^{\alpha} \alpha \mathrm{Sq} \mathrm{L}_{\mathrm{f}}+\mathrm{m}_{\mathrm{y}}^{\omega y} \omega_{\mathrm{y}} S q\left(\mathrm{~L}_{\mathrm{f}}^{2} / \mathrm{V}_{\mathrm{m}}\right) .
\end{aligned}
$$

For simplicity, the following substitutions will be considered.

$$
\begin{aligned}
& a_{1}=\frac{C_{z}^{\alpha} S q}{m V_{m}}, \\
& a_{2}=\frac{m_{y}^{\delta} S q L_{f}}{I_{y y}}, \\
& a_{3}=\frac{m_{y}^{\alpha} S q L_{f}}{I_{y y}}, \\
& a_{4}=\frac{m_{y}^{\omega y} S q L_{f}^{2}}{V_{m} I_{y}},
\end{aligned}
$$

and $a_{5}=\frac{\mathrm{C}_{\mathrm{z}}^{\delta} \mathrm{Sq}}{\mathrm{mV}_{\mathrm{m}}}$.

The physical sense of the introduced coefficients $a_{1}, a_{2}, a_{3}, a_{4}$, and $a_{5}$ is as follows. $a_{1}$ is the coefficient of lifting force by aerodynamic and flow effect. $a_{2}$ and $a_{3}$ are the coefficients of aerodynamic moments due to the control wing and the stabilizing moment of the angle of attack. $a_{4}$ is the coefficient of a damping moment due to an angular speed of bomb rotation around the center of gravity. $a_{5}$ is the coefficient of control fin lifting force. 
Using the above mentioned substitutions, equations (16) and (17) could be written in the following form:

$$
\begin{aligned}
& \dot{\theta}=a_{1} \alpha+a_{5} \delta . \\
& \dot{\omega}_{y}=a_{2} \delta+a_{3} \alpha+a_{4} \omega_{y} .
\end{aligned}
$$

The set of equations (23) and (24) describing the vertical plane motion are used to derive the bomb transfer functions. Using the following relation:

$$
\omega_{y}=\dot{\vartheta} \text {. }
$$

Differentiating equation (5) leads to:

$$
\dot{\alpha}=\dot{\vartheta}-\dot{\theta} \text {. }
$$

From equations (24), (25), and (26)

$$
\omega_{y}=\dot{\alpha}+a_{1} \alpha+a_{5} \delta \text {. }
$$

And from equation (24)

So, $\omega_{y}=\frac{a_{2} \delta(S)+a_{3} \alpha(S)}{S-a_{4}}$

Equations (27) and (28) will be reduced to:

$$
\frac{\alpha(S)}{\delta(S)}=-K_{\alpha} \frac{\left(1+T_{3} S\right)}{\left(T_{2}^{2} S^{2}+2 T_{2} \xi S+1\right)} \text {. }
$$

Where: $K_{\alpha}=\frac{\left(a_{2}+a_{4} a_{5}\right)}{\left(a_{3}+a_{1} a_{4}\right)}$,

$$
\begin{aligned}
T_{3} & =\frac{-a_{5}}{\left(a_{2}+a_{4} a_{5}\right)}, \\
T_{2} & =\frac{1}{\sqrt{-\left(a_{3}+a_{1} a_{4}\right)}},
\end{aligned}
$$

and $\quad \xi=\frac{\left(a_{1}-a_{4}\right)}{2 \sqrt{-\left(a_{3}+a_{1} a_{4}\right)}}$.

From 'equations (25), (27), and (29), the following equation could be obtained:

$$
\frac{\dot{\vartheta}(\mathrm{S})}{\delta(\mathrm{S})}=-\mathrm{K}_{91} \frac{\left[1+\left(\mathrm{T}_{1}+\mathrm{T}_{3}\right) \mathrm{S}+\mathrm{T}_{1} \mathrm{~T}_{3} \mathrm{~S}^{2}\right]}{\left(\mathrm{T}_{2}^{2} \mathrm{~S}^{2}+2 \mathrm{~T}_{2} \xi \mathrm{S}+1\right)}+\mathrm{K}_{92} .
$$

Where: $\quad K_{s 1}=K_{\alpha} a_{1}, \quad T_{1}=\frac{1}{a_{1}}, \quad$ and $\quad K_{92}=a_{5}$.

From equations (23) and (29), the following equation could be written:

$$
\frac{\dot{\theta}(S)}{\delta(S)}=\frac{-K_{\theta 1}\left(1+T_{3} S\right)}{\left(T_{2}^{2} S^{2}+2 T_{2} \xi S+1\right)}+K_{\theta 2}
$$

Where: $\quad K_{\theta 1}=K_{\alpha} a_{1}=K_{91} . \quad$ and $K_{\theta 2}=a_{5}=K_{92}$.

The normal acceleration is given by the following relation:

$$
\mathrm{J}_{\mathrm{N}}=\mathrm{V}_{\mathrm{m}} \dot{\theta} \text {. }
$$

So, $\quad \frac{\mathrm{J}_{\mathrm{N}}(\mathrm{S})}{\delta(\mathrm{S})}=\mathrm{V}_{\mathrm{m}} \frac{\dot{\theta}(\mathrm{S})}{\delta(\mathrm{S})}$, 


$$
\frac{J_{N}(S)}{\delta(S)}=\frac{-K_{\omega 1}\left(1+T_{3} S\right)}{\left(T_{2}^{2} S^{2}+2 T_{2} \xi S+1\right)}+K_{\omega 2} .
$$

Where: $\quad \mathrm{K}_{\omega 1}=\mathrm{V}_{\mathrm{m}} \mathrm{K}_{\theta 1}$ and $\mathrm{K}_{\omega 2}=\mathrm{V}_{\mathrm{m}} \mathrm{K}_{\theta 2}$.

\section{EVALUATION OF BOMB LINEARIZED TRANSFER FUNCTIONS}

The effectiveness of the linearized model and its degree of accuracy will be now considered, the 6-DOF simulation program of the nonlinear model is used in the evaluation of the transient response of bomb airframe alone. During this process, the flight environmental conditions (Mach number and air density) are kept constant. Equations (29), (30), (31), and (32) are the linearized airframe model transfer functions. These equations are used to evaluate the transient response of the airframe due to step input fin deflections. In the mean time, the nonlinear model results are obtained by solving numerically the 6-DOF motion nonlinear differential equations. Runge-Kutta-4 method is utilized with the integration step being chosen such that a good accuracy of calculation is obtained, [7]. The transient response is obtained at different environmental conditions. The results are shown in fig's 4-7.

Inspection of the figures shows that the accuracy of the linearized model in steady state is not affected by the mach number variation. However, it is degraded by the increase of the control fin deflection, as shown in fig (3). This is attributed to the fact that, increasing fin deflection increases the incidence angle and thus the terms considered linear for small incidence and small fin deflection will no longer be linear.

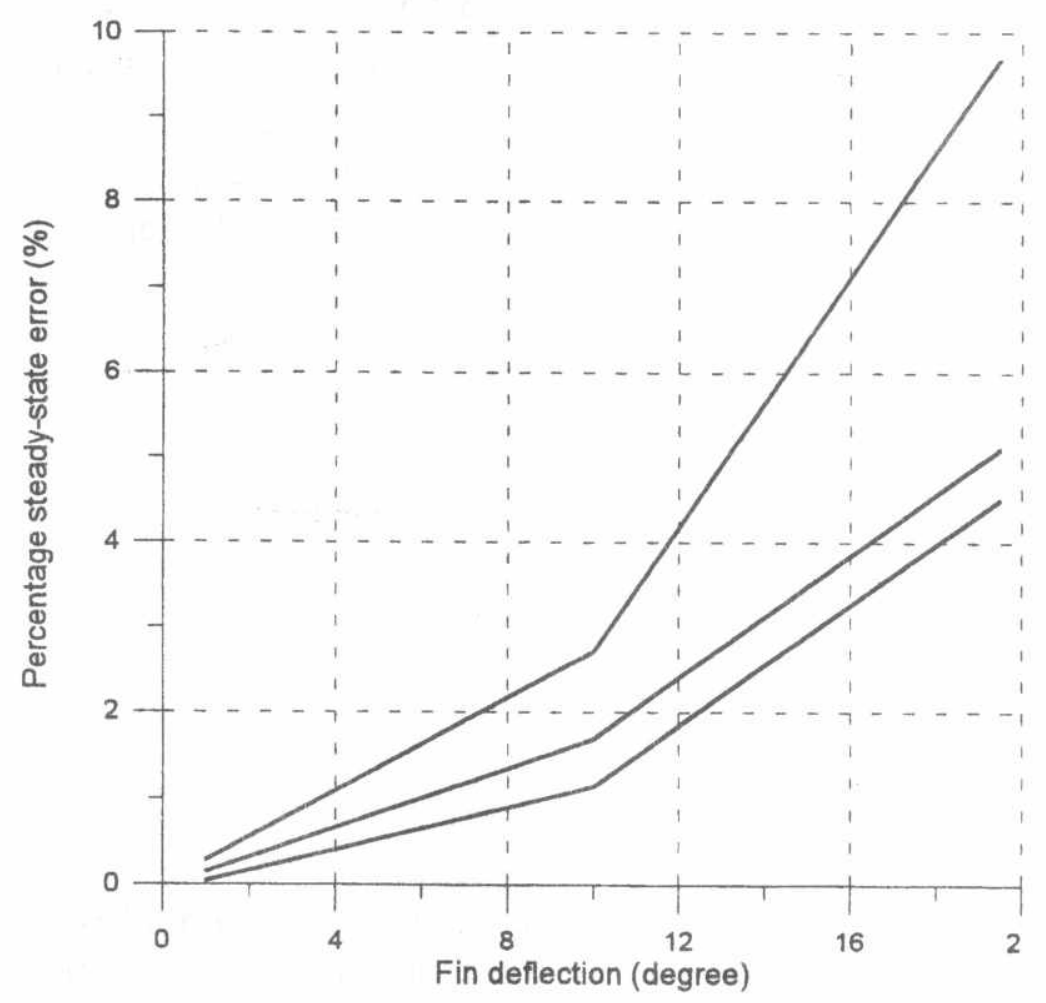


As well, the transient oscillations in the linear model damps faster than that of the nonlinear model which indicated that some of the nonlinear model poles in the left hand side of the S-plane are ignored during the derivation of the linear model equations. In the view of the above results, the linearized model equations given by $29,30,31$, and 32 will be adopted throughout the design of the guidance loop in the coming sections.

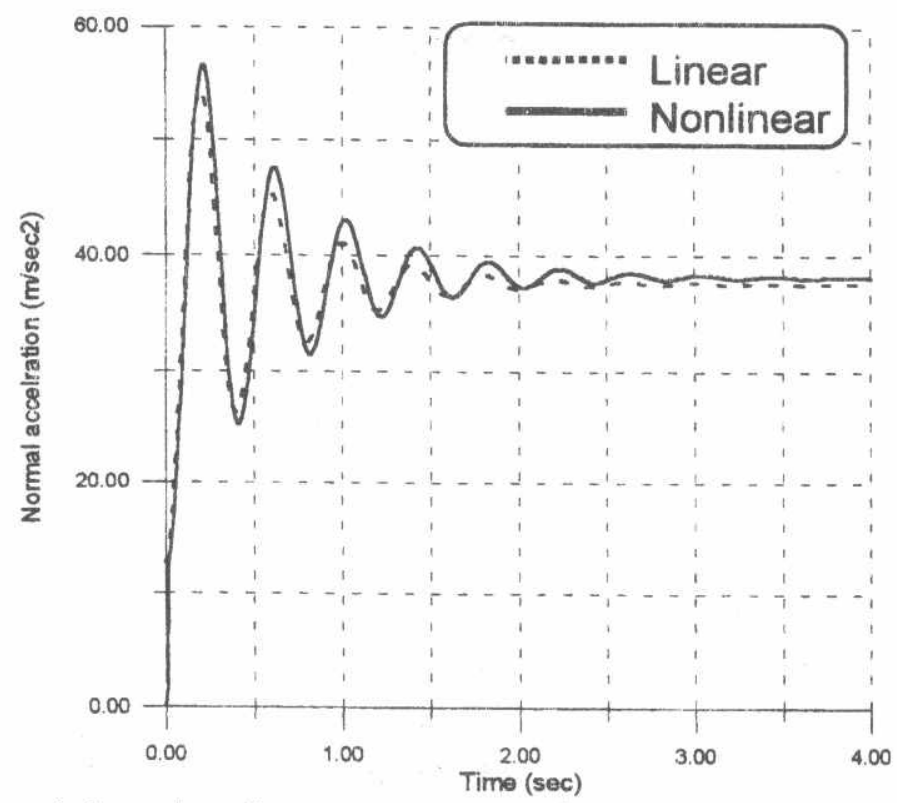

Fig. 4 Acceleration responses of linear and nonlinear models at $\mathrm{M}=0.75$ and $\delta=10^{\circ}$

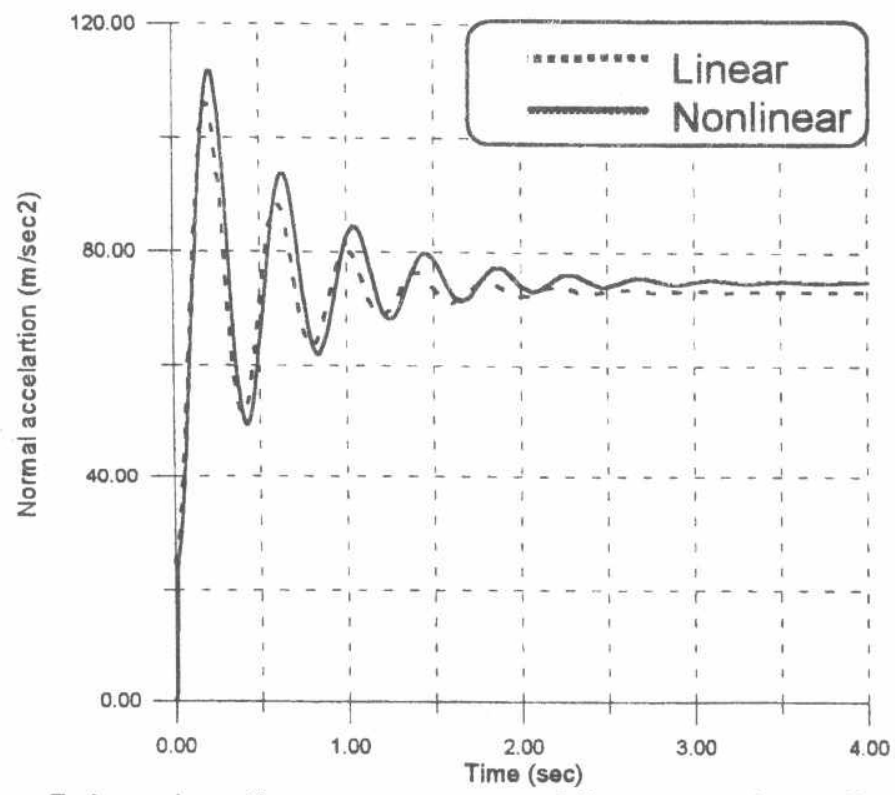

Fig. 5 Acceleration responses of linear and nonlinear models at $M=0.75$ and $\delta=20^{\circ}$ 


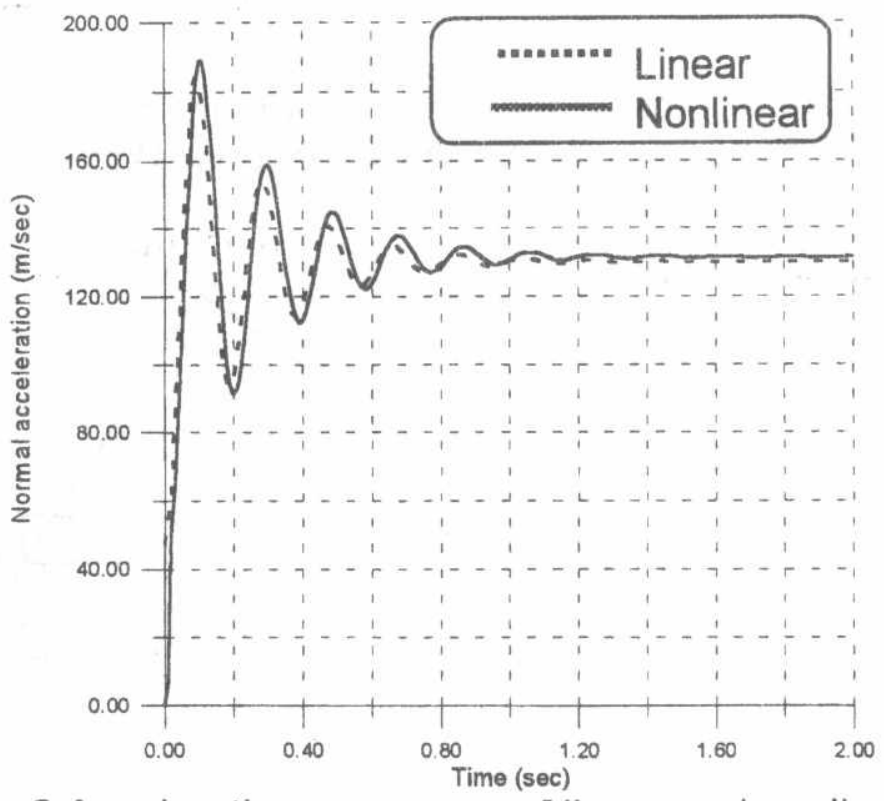

Fig. 6 Acceleration responses of linear and nonlinear models at $\mathrm{M}=1.25$ and $\delta=10^{\circ}$

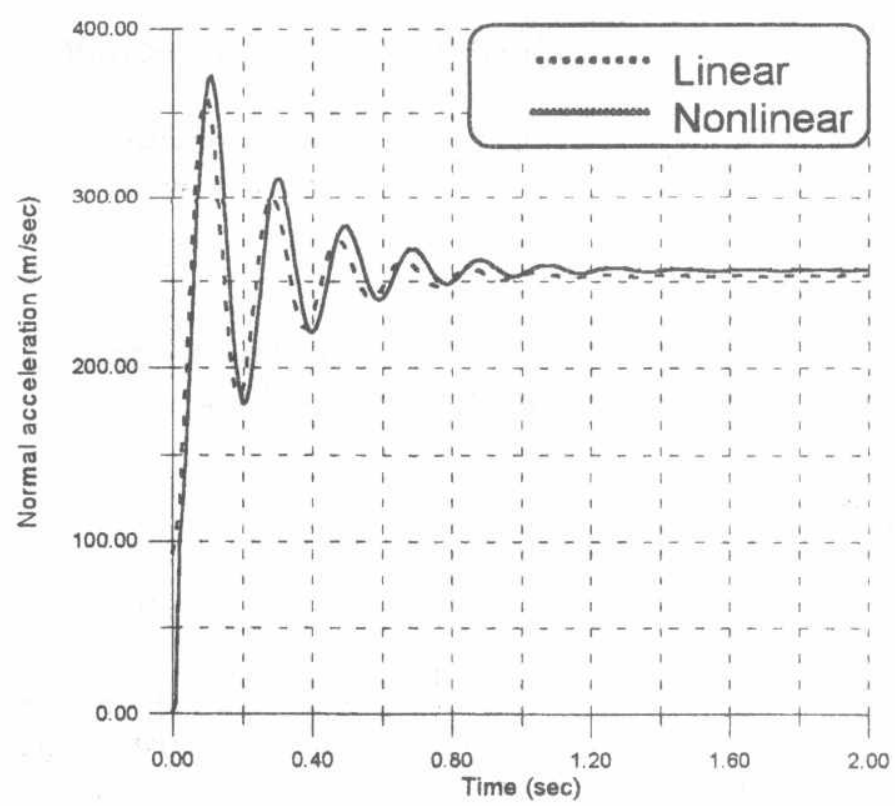

Fig. 7 Acceleration responses of linear and nonlinear models at $\mathrm{M}=1.25$ and $\delta=20^{\circ}$

\section{GUIDANCE LOOP ANALYSIS}

The guidance loop analysis that will be considered is based on the linearized kinematical approach of bomb and target. This assumption is fairly adequate near the end of flight as shown in fig. 8. The intercept position (I) is obtained from the straight line collision courses of target and missile. If at the instant $t_{0}$, the target exerts maneuver given by $Z_{T}$, the bomb will maneuver by $Z_{M}$ and the LOS turns by an angle $\lambda_{p},[5,8]$. 
The aerodynamic transfer functions shown in the model given by fig. 8 is obtained via the 6-DOF model presented by equations (1) to (32).

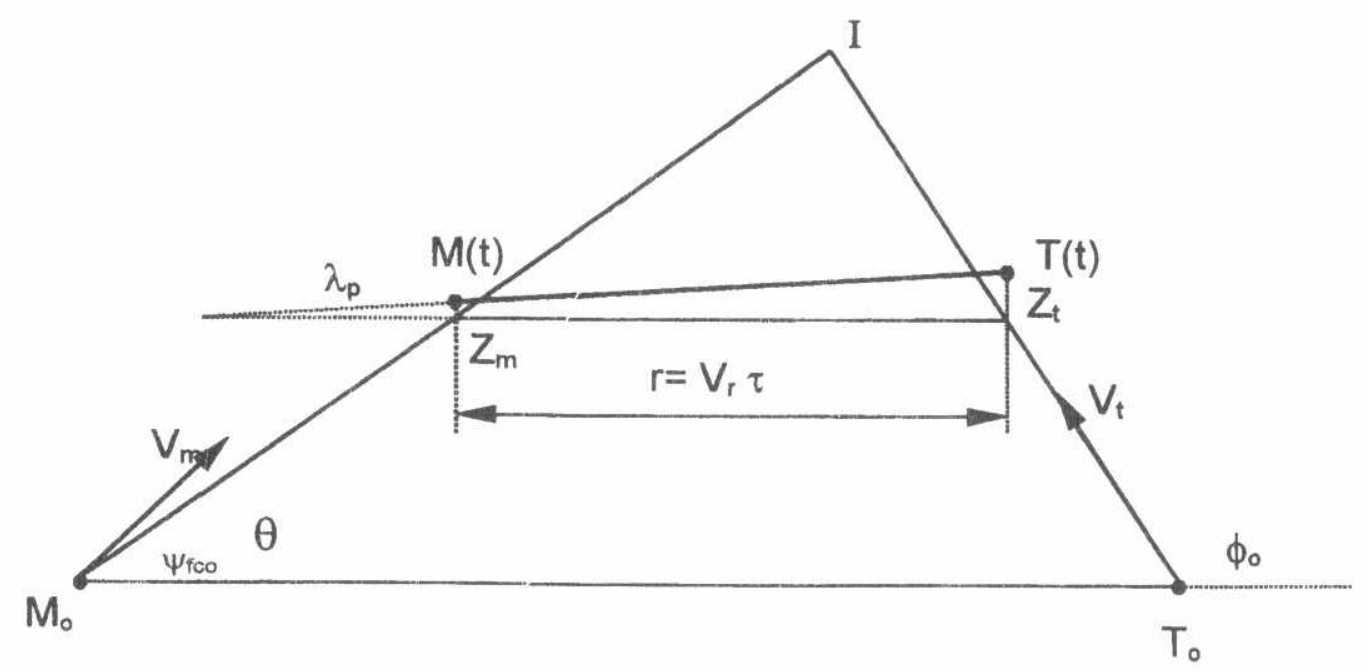

Fig. 8 The bomb-target intercept geometry

The guidance loop is shown in fig. 9. The bomb-target LOS angle as seen by the bomb is given by:

$$
\lambda_{p}=\frac{Z_{T}-Z_{M}}{v_{r} \tau}
$$

$v_{r}$ is the bomb-target closing velocity, $\tau$ is the time-to-go and is given by: $\tau=T-t$. Where $T$ is the total flight time and $t$ is the running time.

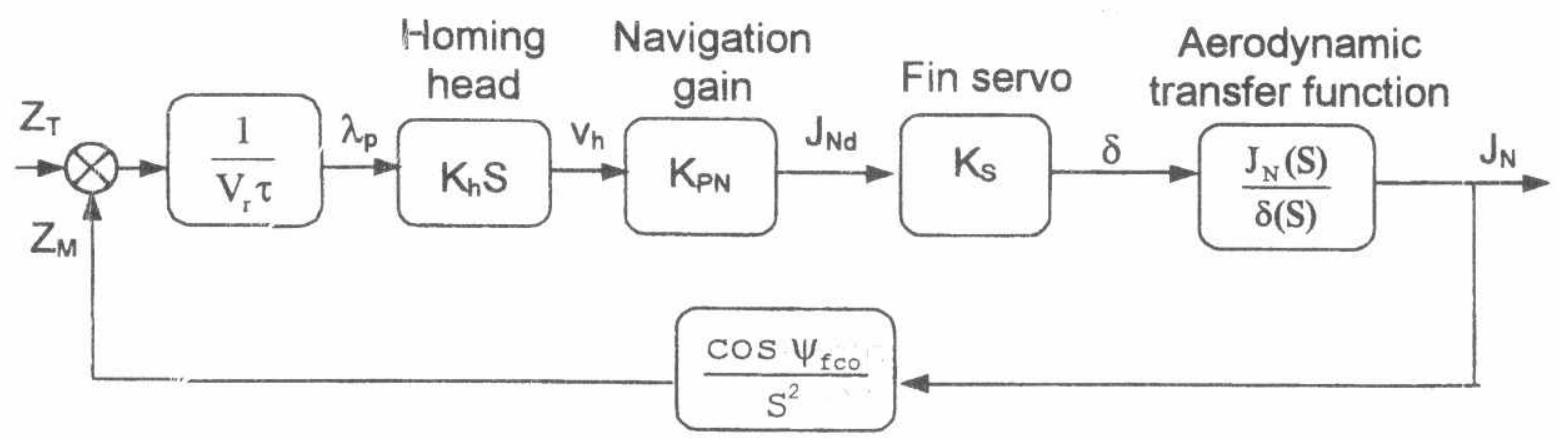

Fig. 9 Block diagram of the guidance loop

Inertialess homing head is considered with gain $K_{h}$ (volt.sec/degree). The horming head produces a voltage $\mathrm{V}_{\mathrm{h}}$ proportional to the bomb-target LOS turn rate $\dot{\lambda}_{p}$. By multiplying the LOS turn rate with the navigation gain $K_{P N}$, the demanded normal acceleration $J_{N d}$ is produced and applied to the fin servo input. The fin servo moves the control fins which forces the bomb to maneuver and deviates in the lateral direction with distance $Z_{m}$. 
Throughout the flight, the time-to-go $\tau$ approaches zero. As well, the bomb speed decreases, which results in time-varying aerodynamic transfer function. Thus, the forward path gain increases, as the bomb approaches the target, which renders the guidance loop unstable beyond some time $t=t_{f}$.

Obviously, well-designed guidance loop should be stable allover the flight time i.e. the closer $t_{f}$ to $T$, the better the guidance loop design. For $t>t_{f}$, the bomb should be close enough to the target such that straight line course results in acceptable missdistance (zero-effort-miss).

In the present situation, the total flight time is $180 \mathrm{sec} . \mathrm{t}_{\mathrm{f}}$ is chosen to be 175 sec. The zero-effort-miss of the bomb after the time $t_{f}$ is calculated and equals 3.15 meters which is an acceptable value. At the time $t_{f}=175 \mathrm{sec}$, the aerodynamic transfer function is obtained according to equation (32) as:

$$
\frac{J_{N}(S)}{\delta(S)}=\frac{0.0045 S^{2}+0.03266 S+6.8}{0.001906 S^{2}+0.011 S+1}
$$

The proportional navigation constant is chosen equals 5 . The fin servo gain is $\left(K_{s}=0.07 \mathrm{rad} / \mathrm{v}\right)$. The homing head gain $K_{h}=5 \mathrm{v} . \mathrm{sec} / \mathrm{deg}\left(K_{h}=286.48 \mathrm{v} . \mathrm{sec} / \mathrm{rad}\right)$. At $t_{f}$ equals $175 \mathrm{sec}$, the distance to go equals 1590 meters and $\operatorname{Cos} \psi_{\mathrm{fco}}=1$. The root locus plot of this system is shown in fig. 10, [9,10].

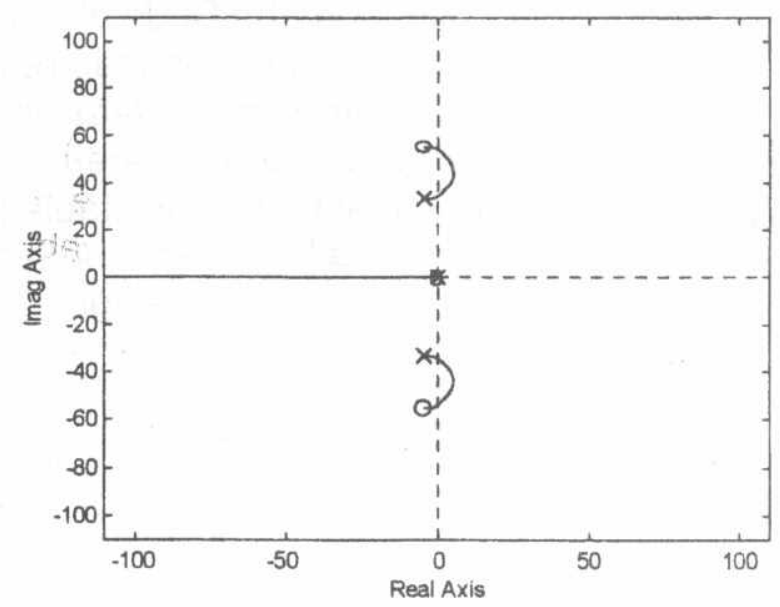

Fig. 10 Root locus plot for the guidance system

It is found from the root locus plot and Routh's stability test that the system is conditionally stable for the following values of the open loop gain $\mathrm{K}[2701.4 \leq \mathrm{K} \leq$ 86.4]. Since, at $t=175 \mathrm{sec}$ the open loop gain of the guidance loop is found to be 4.4 $\mathrm{sec}^{-2}$. Thus, the system at this instant is stable and it continues stable until it reaches a distance equals 81 meters from the target.

In the previous analysis, the homing head is assumed ideal. However, more realistic design requires the inclusion of the finite lags associated with a homing head with second order transfer function. A second order homing head of the following form is used. 


$$
\frac{v_{h}}{\lambda_{p}}=\frac{K_{h} S}{\frac{s^{2}}{\varpi_{n h}^{2}}+\frac{2 \xi_{h}}{\varpi_{n h}}+1}
$$

Where $K_{h}$ is the homing head gain, $\omega_{n h}$ is the natural frequency, and $\xi_{h}$ is the damping ratio of the homing head. The natural frequency is chosen such that $\omega_{n h} . T=100$, where $T$ is the total flight time $(T=180 \mathrm{sec})$; so $\omega_{n h}=0.556 \mathrm{rad} / \mathrm{sec}$. The damping ratio is chosen to be $\xi_{h}=0.8$ and the homing head gain $K_{h}=5 \mathrm{v} / \mathrm{deg}$ $(286.48 \mathrm{v} / \mathrm{rad})$. The block diagram of the guidance loop including the second order homing head at time $t_{f}=175 \mathrm{sec}$ is shown in fig. 11 .

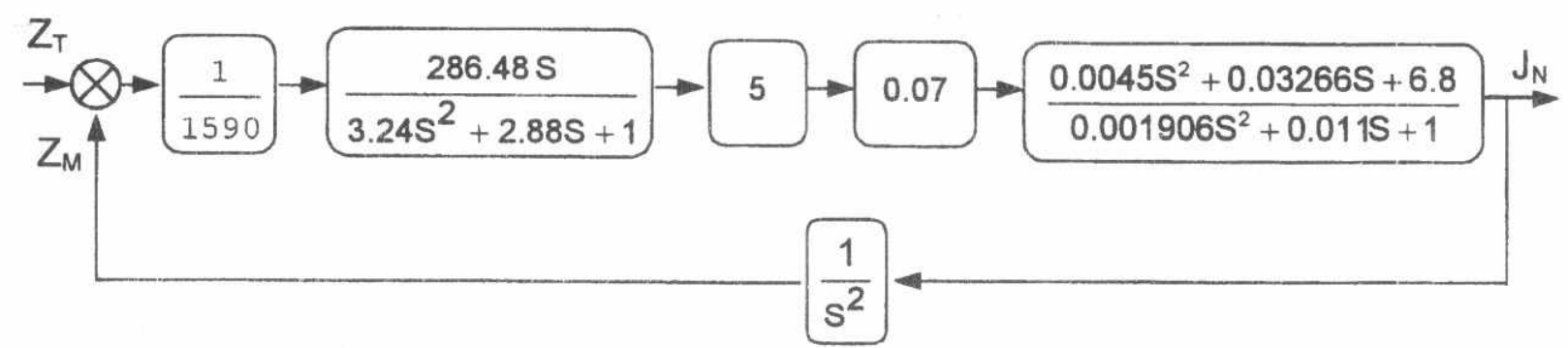

Fig. 11 Block diagram of the guidance loop with second order homing head $\left(\omega_{\mathrm{nh}}=0.556 \mathrm{rad} / \mathrm{sec}\right.$ and $\left.\xi_{\mathrm{h}}=0.8\right)$

The step response of the guidance loop, the Bode plot, and the root locus plot are shown in fig's 12,13, and 14, respectively. The step response shows that the system achieve its steady state after long time ( $t=100 \mathrm{sec})$ with high overshoot. The Bode plot shows that the gain and phase margins are small $(\mathrm{Gm}=2.1 \mathrm{~dB}$ and $\mathrm{Pm}=29^{\circ}$ ). From the root locus plot, the guidance loop is stable for $[\mathrm{K} \leq 0.1249]$ which means that the smallest distance between the bomb and the target beyond which the system goes unstable is $56 \mathrm{Km}$ which is not practical design.

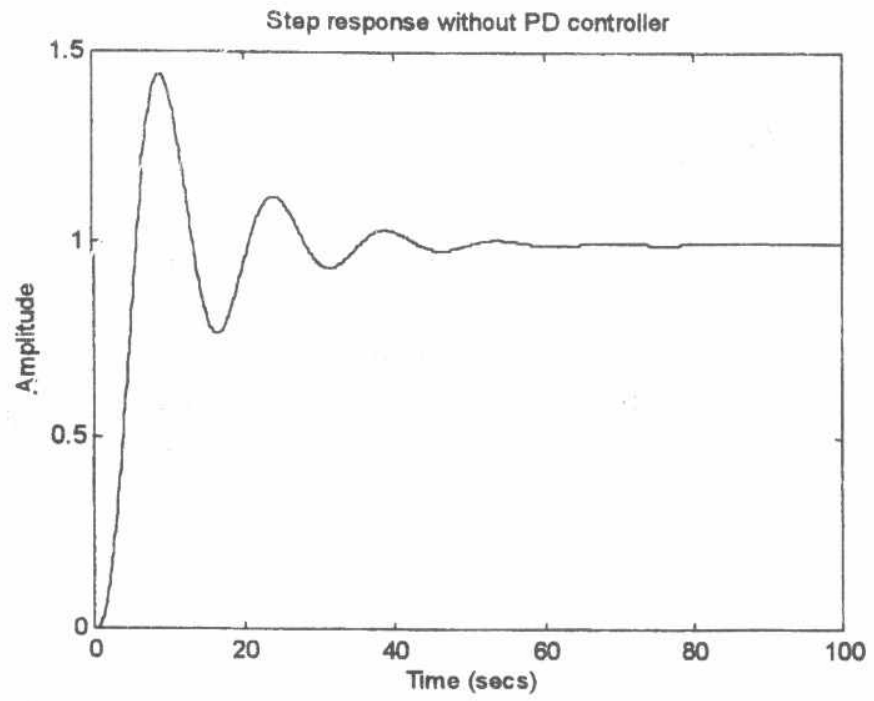

Fig. 12 Step response of the guidance loop 

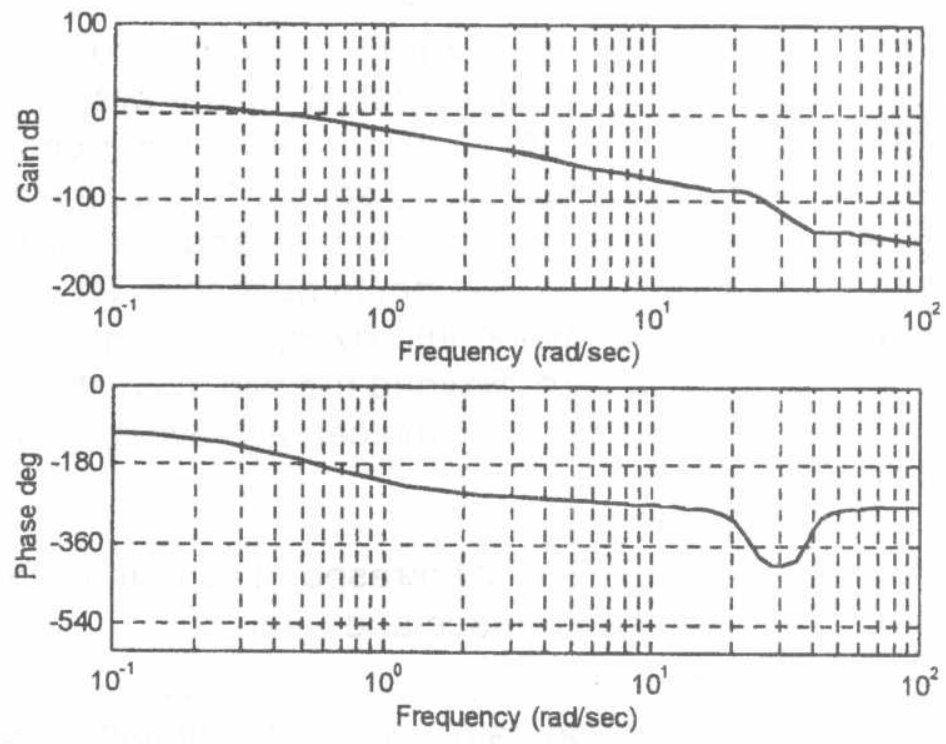

Fig. 13 Bode plot of the guidance loop

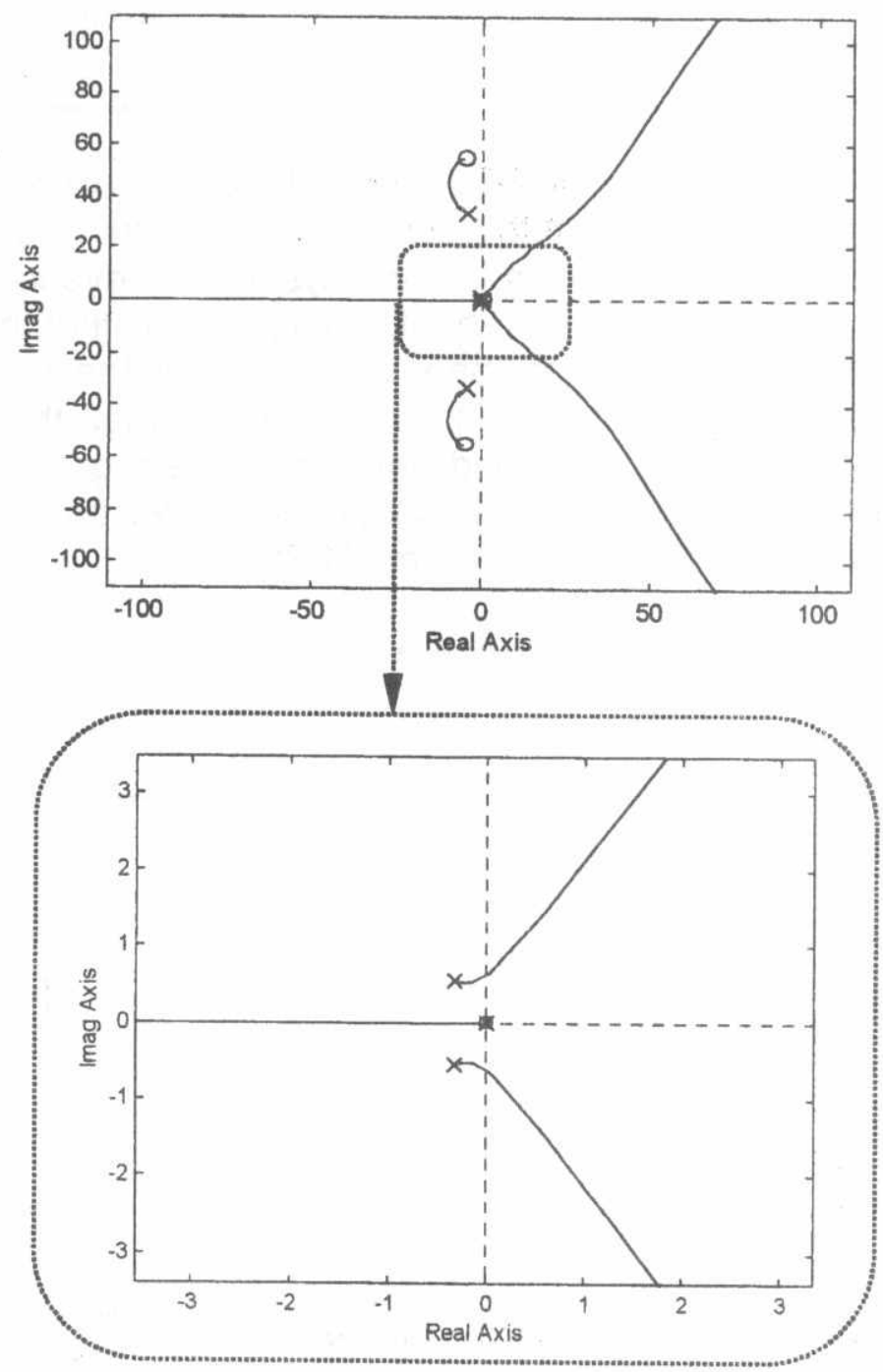

Fig. 14 Root locus plot for the guidance loop 
Thus, it is now necessary to add proportional plus differential controller (PD). Adding this controller to the system adds a zero to the root locus plot which pulls the root locus to the left and adds a margin of stability to the system, [11,12]. The PD controller has the following form $\left(K_{p}+K_{D} S\right)$. Where $K_{p}$ is the proportional factor and equals 1 and $K_{D}$ is the differential factor and equals 10 . So, the zero added to the system is $(S+0.1)$. Table 1 shows the location of zeros and the corresponding limit of gain $\mathrm{K}$ and minimum LOS range for the system to be stable. The obtained minimum LOS range values indicate that a major improvement in the system performance could be achieved by adding this zero. Beyond the minimum stable LOS range, the guidance system can be stopped and the bomb can achieve its mission successfully through the straight line course.

Table 1 Zero locations and corresponding gain limits $\left(\omega_{\mathrm{nh}}=0.556 \mathrm{rad} / \mathrm{sec}\right.$ and $\left.\xi_{\mathrm{h}}=0.8\right)$

\begin{tabular}{|c|c|c|}
\hline Zero location & Open loop gain & Min. stable LOS range \\
\hline-0.1 & $\mathrm{~K} \leq 40.87$ & $\mathrm{r}=17.1 \mathrm{~m}$ \\
\hline-0.2 & $\mathrm{~K} \leq 33.59$ & $\mathrm{r}=41.7 \mathrm{~m}$ \\
\hline-0.3 & $\mathrm{~K} \leq 25.55$ & $\mathrm{r}=82.1 \mathrm{~m}$ \\
\hline
\end{tabular}

The step response, the root locus plot, and the Bode plot of the guidance loop with the PD controller with zero at $(-0.1)$ are shown in fig's 15,16 , and 17 , respectively. The step response shows that the system achieve its steady state after short time $(t=30 \mathrm{sec})$ with small overshoot, which means that the response of the system with PD controller is faster than before. From the root locus plot, the guidance loop is stable for [ $\mathrm{K} \leq 40.87]$ which means that the smallest distance between the bomb and the target beyond which the system goes unstable is $17.1 \mathrm{~m}$ which is acceptable for practical use. The Bode plot shows that the gain and phase margins are increased $\left(\mathrm{Gm}=63.6 \mathrm{~dB}\right.$ and $\left.\mathrm{Pm}=41.7^{\circ}\right)$.

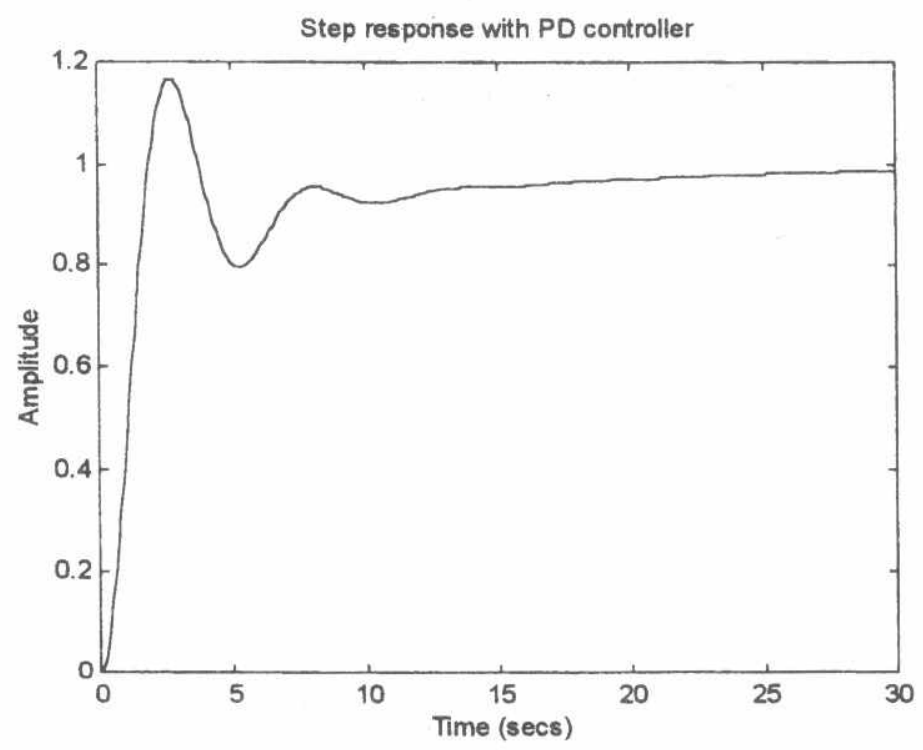

Fig. 15 Step response of the guidance loop with PD controller 

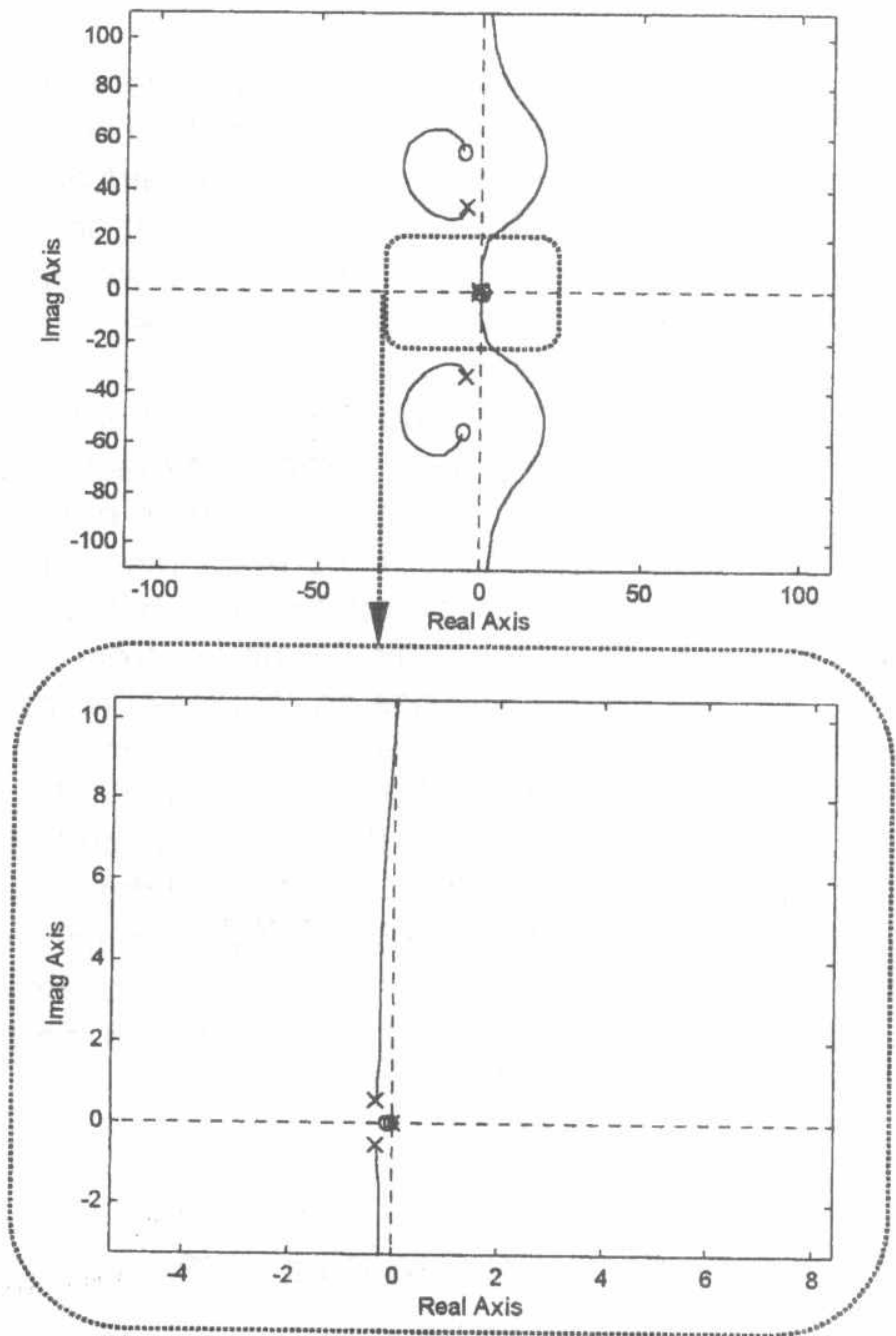

Fig. 16 Root locus plot for the guidance loop with PD controller
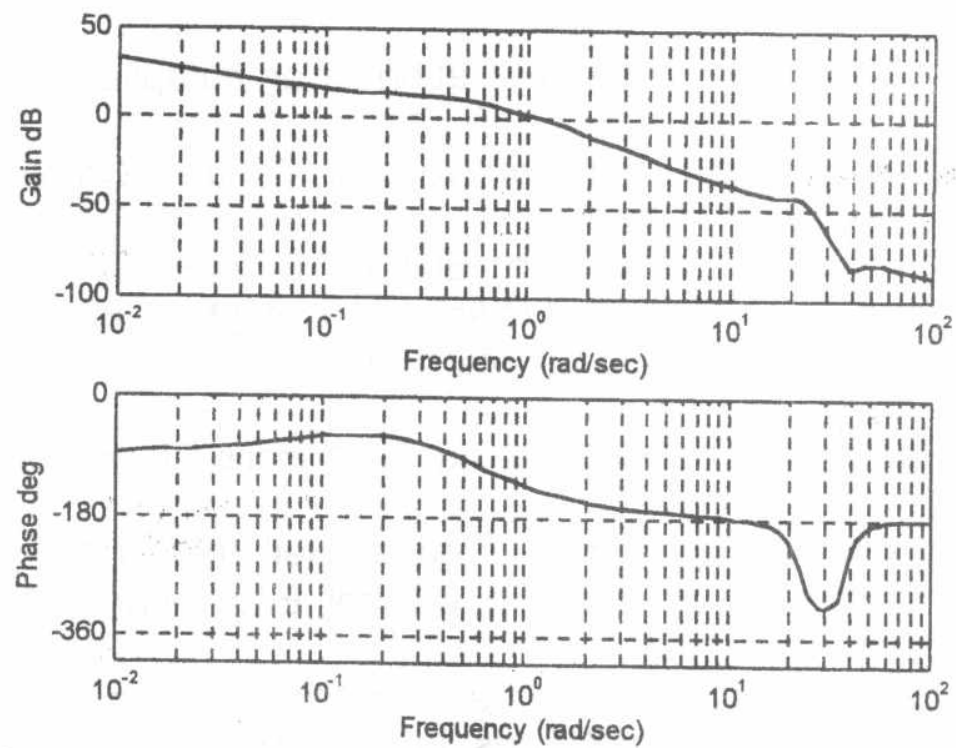

Fig. 17 Bode plot of the guidance loop with PD controller 


\section{CONCLUSIONS:}

The design of a new guidance system or the improvement of an existing system requires a complete information about the main building blocks of the system. Thus, the obtained nonlinear aerodynamic model of the bomb airframe was linearized to obtain the transfer function of the bomb airframe at different points of the trajectory. A comparison between linear and nonlinear models shows that, there is a slight difference between the linear model and the nonlinear model responses. The steady state error increases as fin deflection increases for different bomb speeds. As well, the transient oscillations in the nonlinear model damps faster than that of the linear model. For the bomb of concern, the values of the fin deflection and the incidence angle are small enough to make linear model results close to nonlinear results.

The stability of guidance loop of the bomb is an important requirement. The designed guidance loop is conditionally stable for ideal homing head. The system will be stable until it reaches a distance equals 81 meters from the target and this is not a good value. However, more realistic design requires the inclusion of the finite lags associated with the homing head. So, a second order homing head is used. In this case, it is necessary to add proportional plus differential (PD) controller. Adding this controller to the system adds a zero to the root locus plot which pulls the root locus to the left and increases the stability margins of the system. The obtained minimum LOS range is 17.1 meters, which indicates that a major improvement in the system performance could be achieved by adding this zero. Beyond the minimum stable LOS range, the guidance system can be stopped and the bomb can achieve its mission successfully through the straight line course.

\section{REFERENCES:}

[1] Eric H. Biass, "Aircraft Armament", Interavia Data, (1984).

[2] R. T. Davis, Smart Bombs Perform Interdiction Surgery, Microwaves, (1972).

[3] R. Goodstein, Terminal Guidance Systems, AGARD Lecture Series NO. 52, (1972).

[4] A. S. Gad, Msc, Design and experimentation for an autopilot for guided bombs, (1998).

[5] P. Garnell and D.J. East, Guided Weapon Control Systems, $2^{\text {nd }}$ edition, Pergamon press, New York, (1980).

[6] A. M. Wagdy, Msc, Analysis of Stabilizing Effect of Different Types of Gyroscopes on Different Configurations of Guided Missiles Autopilot, (1980).

[7] E. Heap, Numerical Analysis and Simulation Evolution, AGARD Lecture Series, No. 52 , (1972).

[8] R. Goodstein, Guidance Law Applicability For Missile Closing, AGARD Lecture Series NO. 52, (1972).

[9] K. Ogata, Modern Control Engineering, Prentice-Hall, Inc., (1985).

[10] R. C. Drof, Bishop R. H., Modern Control Engineering, Addison-Wesley Publishing Company, Inc., (1995).

[11] H.B. John, Automatic Control of Aircraft And Missiles, 2nd edition, Institute for Aerospace Studies, John Willey \& Sons, Inc., (1991).

[12] B. Etkih, Dynamics of flight stability and control, 2nd ed., Institute For Aerospace Studies, John Willey \& Sons, (1982). 